\title{
ICD 10 GM: DIMDI aktualisierte Dekubitusklassifikation
}

\author{
Gemeinsame Sprache für alle Gesundheitsberufe möglich
}

Mit Wirkung zum 1. Januar 2010 hat das Deutsche Institut für medizinische Dokumentation und Information - kurz DIMDI - im Rahmen der routinemäßigen Aktualisierung aufgrund der internationalen englischsprachigen WHO-Fassung der ICD 10 eine Änderung des Kode L.89 = Kodierung des Dekubitus - vorgenommen. Die Kodierung nach ICD 10 GM entspricht nun der international gängigen Kodierung unter Bezug auf die Klassifikation nach EPUAP 1998 in Übersetzung des DQNP 2008 und basiert somit auf pflegewissenschaftlichen Erkenntnissen. Somit ist der Kode L89 pflegerelevant und bietet vor allem die Grundlage für eine gemeinsame Sprache aller Berufe im Gesundheitswesen. Die Änderungen im Einzelnen:
(Bisher: Tiefer Hautdefekt, Muskeln und Sehnen sind sichtbar und eventuelle betroffen.)

- Grad 4 (L89.3) ist definiert als: „Ausgedehnte Zerstörung, Gewebsnekrose oder Schädigung von Muskeln, Knochen oder stützenden Strukturen, mit oder ohne Verlust aller Hautschichten."

(Bisher: Tiefer Hautdefekt mit Knochenbeteiligung).

Aus den Definitionen ergeben sich die wesentlichen Änderungen: Bisher war der Grad 4 ausschließlich durch die Knochenbeteiligung definiert. Nunmehr entspricht bereits eine Muskelbeteiligung dem Grad 4 mit der Folge, dass ein bisher als Grad 3 eingestufter Dekubitus zukünftig als Dekubitus Grad 4 eingestuft werden kann.

Hinzuweisen ist, dass jeweils der nied-

\section{Die Übernahme pflegewissenschaftlich begründeter, interna-} tional anerkannter Definitionen bietet die große Chance eine gemeinsame Sprache zu finden.

Die Schlüsselnummern beginnen mit 0 statt wie bisher mit 1. Das heißt, der Dekubitus 1. Grades wird nunmehr als L89.0 verschlüsselt. Entsprechend ist

- Grad 1 (L89.0) definiert wie folgt:

„Nicht wegdrückbare Rötung intakter Haut. Besonders bei dunkelhäutigen Menschen können auch Hautverfärbungen, Überwärmung, Ödem oder Verhärtung Indikatoren für Stadium 1 sein."

(Bisher: Umschriebene Rötung bei intakter Haut).

- Grad 2 (L89.1) ist definiert als: „Teilverlust der Haut mit Schädigung von Epidermis, Dermis oder beiden Hautschichten. Das Druckgeschwür ist oberflächlich und manifestiert sich klinisch als Hautabschürfung oder Blase." (Bisher: Hautdefekt)

- Grad 3 (L89.2) ist definiert als: „Verlust aller Hautschichten einschließlich Schädigung oder Nekrose des subkutanen Gewebes, die bis auf, aber nicht unter die Faszie reichen kann." rigere Grad zu kodieren ist, wenn der Grad eines Dekubitalgeschwüres nicht sicher bestimmt werden kann.

\section{Praktische Konsequenz für die Pflege}

Unabhängig von der Frage, ob Pflegepersonen für die Pflegedokumentation die Kodierung, die auch Ziffern für Lokalisation enthält (s. Kasten), übernehmen - was

\begin{tabular}{|ll|}
\hline $\begin{array}{l}\text { Lokalisation } \\
\text { ko kodiert }\end{array}$ & Kopf \\
\hline $1=$ & Obere Extremität \\
\hline $2=$ & Dornfortsätze \\
\hline $3=$ & Beckenkamm, Spina iliaca \\
\hline $4=$ & Kreuzbein, Steißbein \\
\hline $5=$ & Sitzbein \\
\hline $6=$ & Trochanter \\
\hline $7=$ & Ferse \\
\hline $8=$ & $\begin{array}{l}\text { Sonstige Lokalisationen der unteren } \\
\text { Extremität }\end{array}$ \\
\hline $9=$ & Sonstige und nicht näher bezeichnete \\
\hline $\begin{array}{l}\text { Ein Beispiel: } \text { Ein Fersendekubitus } 2 . \text { Grades am } \\
\text { rechten Bein könnte in der Dokumentation } \\
\text { festgehalten werden mit: } \text { L89.1.7 rechts. }\end{array}$ \\
\hline $\begin{array}{l}\text { Zur Beschreibung der Lokalisation sind bei der Kategorie L89 } \\
\text { hinter der 4. Stelle = Grad die voraufgeführten fünften Stellen zu } \\
\text { benutzen. }\end{array}$ \\
\hline
\end{tabular}

übrigens auch die Pflegearbeit sehr vereinfachen würde, da alle Beteiligten wissen, was dem Ziffernkode hinterlegt ist, bietet die Übernahme pflegewissenschaftlich begründeter, international anerkannter Definitionen die große Chance für alle Beteiligten, eine gemeinsame Sprache zu finden.

\section{Korrespondenzanschrift: Heidi Heinhold Mitglied Deutsche Dekubitusliga e.V. Zeithstrasse 5 \\ D-51766 Engelskirchen \\ Tel. 0049/2263 - 951380 \\ Fax: 0049/2263 - 95138 \\ E-Mail: Heinhold.Heidi@t-online.de \\ Internet: www.ppmr-redaktion-heinhold.de}

\section{Buchempfehlung}

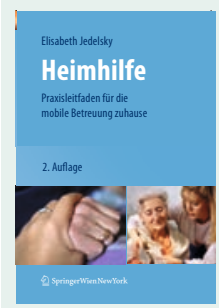

\section{Heimhilfe}

Praxisleitfaden für die mobile Betreuung zuhause

Jedelsky, Elisabeth (Hrsg.)

2., korr. u. erw. Aufl., Springer WienNewYork 2010,

256 S. 7 Abb., Softcover

ISBN: 978-3-211-99226-5,

Preis: $29,95 €$

Autoren mit jahrelanger Erfahrung verfassten dieses praxisnahe Buch. Zusätzlich sind die aktuellsten Änderungen im Heimhilfegesetz eingearbeitet. 